\title{
Effects of Ras homolog gene family, member $C$ gene silencing combined with rapamycin on hepatocellular carcinoma cell growth
}

\author{
SHU-LI XIE ${ }^{1}$, MING-GUANG ZHU², GUO-FU CHEN ${ }^{1}$, GUANG-YI WANG ${ }^{1}$ and GUO-YUE LV ${ }^{1}$ \\ ${ }^{1}$ Department of General Surgery, First Hospital of Jilin University; ${ }^{2}$ Department of Immunology, \\ Basic Medical College of Jilin University, Changchun, Jilin 130021, P.R. China
}

Received August 14, 2014; Accepted June 16, 2015

DOI: $10.3892 / \mathrm{mmr} .2015 .4056$

\begin{abstract}
The aim of the present study was to investigate the combined effects of inhibiting the Ras homolog gene family, member C (RhoC)/Rho kinase and phosphoinositide 3 kinase/Akt/mammalian target of rapamycin (mTOR) pathways on hepatocellular carcinoma cell growth. The RhoC gene was silenced by RNA interference (RNAi) and mTOR was inhibited by rapamycin (RAPA). Subsequently, an MTT assay for cell growth detection, western blot analysis for gene expression analysis, silver nitrate staining for cell proliferation, Wright's staining for analysis of the apoptotic rate analysis, soft agar clonogenic assay for the determination of cell growth characteristics and a Transwell assay for cell migration were performed. RhoC expression in hepatoma cell lines was lower than that in the HL7702 normal human liver cell line. The level of cell proliferation in the RNAi + RAPA group was lower than that in the RNAi, RAPA and Scramble groups. The levels of cyclin-dependent kinase 2 in the RNAi + RAPA group were lower than those in the other groups, while the levels of P16 in the RNAi + RAPA group were higher than those in the other experimental groups. No significant difference was found between the RNAi + RAPA and the normal HL7702 group. The number of silver nitrate-stained particles was reduced in the RNAi + RAPA group compared with that in the other groups. No significant difference was found between the RNAi + RAPA and HL7702 groups. Wright's staining for apoptosis demonstrated that apoptosis in the Scramble group was rare, while the RAPA and RNAi groups contained a large number of apoptotic cells, which displayed nuclear condensation, fragmentation, deepened staining, as well as a wrinkled
\end{abstract}

Correspondence to: Dr Guo-Yue Lv, Department of General Surgery, First Hospital of Jilin University, 71 Xinmin Street, Changchun, Jilin 130021, P.R. China

E-mail: xieshuli6310@hotmail.com

Key words: hepatocellular carcinoma, gene silencing, rapamycin, Ras homolog gene family, member C, phosphoinositide 3 kinase/AKT/mammalian target of rapamycin membrane. B-cell lymphoma-2 (Bcl-2) expression in the $\mathrm{RNAi}+\mathrm{RAPA}$ group was lower than that in the other groups, while the gene expression of Bcl-2-associated X protein in the RNAi + RAPA group was increased compared with that in the other groups. No cell colony formation was observed in the soft agar cloning experiment in the RNAi + RAPA and HL7702 group, while in the other groups, visible cell clones appeared. In the Transwell assay the number of migrated cells in the RNAi + RAPA group was lower than that in the other groups. The gene expression of matrix metalloproteinase (MMP)2, MMP-9 and vascular endothelial growth factor in the RNAi + RAPA group was lower than that in the other experimental groups. In conclusion, RhoC gene silencing combined with RAPA was able to significantly inhibit the growth of hepatocellular carcinoma cells.

\section{Introduction}

Liver cancer is one of the most lethal malignancies with a clinically poor prognosis. There are two main reasons for this poor prognosis: One is the difficulty in the early detection of liver cancer. Patients with clinical symptoms are already at an advanced cancer stage, meaning that they have already missed the chance for surgical treatment. The second is the current lack of effective chemotherapeutic treatment for patients with liver cancer. No drug treatments are currently available for patients with liver cancer (1). In recent years, with a greater understanding of the molecular mechanisms of cancer, targeted therapy has become a novel concept for tumor treatment. Targeted therapy achieves its therapeutic purposes by means of targeting those molecules, which are important in tumor growth, invasion or metastasis. The majority of common targets are the critical molecules in cell signaling pathways, and interfering with these molecules may block the respective signaling pathway and lead to tumor growth inhibition or apoptosis (2).

The association between Ras homolog gene family, member C (RhoC) overexpression and tumor development has become an intense area of interest in recent years. A growing number of investigations have utilized RhoC as a target molecule for cancer gene therapy (3-5). RhoC has a marked impact on local tumor cell invasion and distant metastasis through 
regulation of the actin cytoskeleton. Its elevated expression in hepatoma cells promotes tumor invasion and distant metastasis (6). In addition, RhoC overexpression is also an important factor in promoting hepatoma cell proliferation (7). RhoC also induces malignant transformation of normal liver cells (8). Therefore, RhoC overexpression is essential for the survival, invasion and metastasis of hepatocellular carcinoma cells. Overexpression of RhoC is also important in the invasion and distant metastasis of liver cancer cells. RhoC as a molecular target is expected to produce good results for hepatocellular carcinoma treatment.

However, the mechanisms of tumor cell survival and invasion are complex, and specific control of the growth and metastasis of tumor cells is difficult. Therefore, researchers have begun to adopt a joint approach to eradicate or inhibit the growth of tumor cells. The phosphoinositide 3 kinase (PI3K)/Akt/mammalian target of rapamycin (mTOR) signal transduction pathway is closely associated with tumor cell proliferation, invasion and apoptosis, and has thus become an area of interest in the study of liver cancer (9). Rapamycin (RAPA) is a well-known specific inhibitor of the PI3K/Akt/mTOR signaling pathway. Thus, inhibition of mTOR activity may lead to tumor cell growth suppression.

RAPA does not act directly on the mTOR molecule. It first binds with the FK506 protein to form a complex. This complex attaches with the FRB domain of mTOR, disturbing mTOR signaling, affecting the synthesis of associated proteins, and therefore inhibiting mTOR activity (10). RAPA has potent clinical effects against cervical cancer, endometrial cancer and ovarian cancer (11). Its derivatives, including letrozole and everolimus, are able to prohibit the growth and progression of breast cancer, kidney cancer and pancreatic cancer, respectively (11-13). For patients with liver cancer, RAPA and its derivatives may also have a good therapeutic prospect. In addition to the direct inhibition of tumor cell proliferation, RAPA also inhibits the proliferation of vascular endothelial growth factor (VEGF) and vascular endothelial cells, which are important in cancer therapy $(14,15)$. It is known that the RhoC and PI3K/Akt/mTOR pathways are closely linked (13). RhoC overexpression leads to PI3K/Akt/mTOR pathway activation in melanoma cells, thus promoting melanoma metastasis (16). Following PI3K/Akt/mTOR pathway activation, the activated Akt molecule phosphorylates RhoC, promoting breast cancer cell metastasis (17). Thus, the RhoC and the PI3K/Akt/mTOR pathway are able to activate each other and are important in tumor growth. Therefore, it was hypothesized that the joint effort of targeting RhoC and the PI3K/Akt/mTOR pathway may produce synergistic effects in liver cancer treatment. Therefore, the present study selected hepatocellular carcinoma cells to investigate the combined effects of RhoC gene silencing and the PI3K/Akt/mTOR pathway-specific inhibitor RAPA on the biological behavior of hepatocellular carcinoma cells, including proliferation, invasion and migration, providing theoretical and experimental evidence for liver cancer treatment.

\section{Materials and methods}

Reagents and antibodies. pU6mRFP RhoC-small interfering (si)RNA and pU6mRFP scramble-small interfering (si)RNA plasmids were constructed in our laboratory (Department of General Surgery, First Hospital of Jilin University, Changchun, China). The DNA molecular weight standard DL2000 and the reverse transcriptional polymerase chain reaction (RT-PCR) kit (RNA PCR kit version 3.0) were purchased from Takara Biotechnology Co.,Ltd.(Dalian,China).SuperFect Transfection Reagent (cat. no. 301305) was purchased from Qiagen China Co., Ltd. (Shanghai, China). RAPA was purchased from LC Laboratories (Woburn, MA, USA). The primary mouse anti-human $\beta$-actin antibody (cat. no. MA5-15739; 1:500) was purchased from Invitrogen Life Technologies (Carlsbad, CA, USA), goat anti-mouse secondary antibody (cat. no. sc-2005; 1:500), goat anti-rabbit secondary antibody (cat. no. sc-2030; 1:500) and rabbit anti-human RhoC polyclonal antibody (cat. no. sc-28565; 1:200).

Cell culture and transfection. BEL7402 human hepatoma cells were purchased from Shanghai Institute of Biochemistry and Cell Biology Cell Bank (Shanghai, China). The cells were cultured at $37^{\circ} \mathrm{C}$ in 4-(2-hydroxyethyl)-1-piperazineethanesulfonic acid-buffered Dulbecco's modified Eagle's medium (H-DMEM; Invitrogen Life Technologies) in a $5 \% \mathrm{CO}_{2}$ incubator (Shanghai Shen-Li High Tech Co. Ltd., Shanghai, China). Cells in the logarithmic growth phase were seeded into 24 -well plates until the cell density reached $\sim 70 \%$ confluence. Transfection was performed according to the manufacturer's instructions. Cells were cultured in an incubator following transfection, and the culture medium was discarded $4 \mathrm{~h}$ later. Cells were washed with phosphate-buffered saline (PBS) solution, and $400 \mu \mathrm{l}$ fresh H-DMEM containing $10 \%$ fetal bovine serum, $1 \%$ penicillin and $1 \%$ streptomycin (all Invitrogen Life Technologies) was added to each well.

BEL7402 cells were used as the blank control group, BEL7402 cells transfected with pU6mRFP scramble-siRNA were used as the negative control group, and BEL7402 cells transfected with pU6mRFP RhoC-siRNA were used as the RNA interference (RNAi) group. Following $72 \mathrm{~h}$ of incubation, $1 \times 10^{6}$ cells/ml were harvested and lysed with TRIzol reagent (Invitrogen Life Technologies) for total RNA extraction. RNA was then reverse transcribed to cDNA, according to the manufacturer's instructions. Semi-quantitative RT-PCR was performed to detect RhoC mRNA expression levels. PCR was conducted using the RNA PCR kit version 3.0 and a PCR Thermal Cycler Dice (Takara Biotechnology Co., Ltd.). The primer sequences were as follows: RhoC upstream, 5'-ATG GCTGCAATCCGAAAGAAG-3' and downstream, 5'-TCA GAGAATGGGACAGCCCCT-3'. Tubulin upstream, 5'-CAC CCGTCTTCAGGGCTTCTTGGTTT-3' and downstream, 5'-CATTTCACCATCTGGTTGGCTGGCTC-3' (Sangon Biotech Co., Ltd., Shanghai, China). The PCR reaction mixture $(50 \mu \mathrm{l})$ consisted of: $1 \mu \mathrm{l}$ cDNA, $10 \mu \mathrm{l}$ 5X PCR buffer, $1 \mu \mathrm{l}$ upstream primer, $1 \mu \mathrm{l}$ downstream primer, $4 \mu \mathrm{l}$ $\mathrm{MgCl}_{2}, 1 \mu \mathrm{l}$ mixed dNTP, $31.5 \mu \mathrm{ldd} \mathrm{dd}_{2} \mathrm{O}$, and $0.5 \mu \mathrm{l}$ Ex Taq DNA polymerase. The annealing temperature was $57^{\circ} \mathrm{C}$, and 30 cycles were set for RhoC amplification and 25 cycles for tubulin amplification. Tubulin was used as a housekeeping gene. Agarose gel electrophoresis was conducted, then grayscale analysis was performed using the Image Master Analysis system. The following equation was used to calculate the inhibition ratio: Inhibition ratio $=$ (grayscale in the control 
group - grayscale in the experimental group) / grayscale in the control group x $100 \%$.

Western blot analysis for protein determination. The cells were lysed with protein lysis buffer (Watson Biotechnology Co., Ltd., Beijing, China) and RhoC protein was determined through western blot analysis. The total cell protein sample obtained from the lysed cells was loaded onto wells (30 $\mu \mathrm{g}$ protein for each lane) for SDS-PAGE. After 12\% SDS-PAGE at $120 \mathrm{~V}$, transmembrane electrophoresis onto a polyvinylidene difluoride membrane (GE Osmonics, Inc., Minnetonka, MN, USA) was performed for 1.5-2 h under constant electrical flow, with the electrical current $(\mathrm{rnA})=$ gel area $\mathrm{x} 2$. The membrane was blocked with $5 \%$ skimmed milk powder overnight. Subsequently, the membrane was probed with primary antibodies at $37^{\circ} \mathrm{C}$ for $2 \mathrm{~h}$. The membrane was then washed three times (10 $\mathrm{min} /$ wash) with tris-buffered saline containing $0.05 \%$ (v/v) Tween 20 (TBST; Sigma-Aldrich, St. Louis, MO, USA), followed by $1.5 \mathrm{~h}$ incubation at $37^{\circ} \mathrm{C}$ with secondary antibodies. The membrane was subsequently washed a further three times with TBST and the antibodies were visualized using an enhanced chemiluminescence (ECL) kit (LumiPico ECL kit; Shanghai ShineGene Molecular Biotech, Inc., Shanghai, China), and the membrane was exposed to X-ray irradiation according to the manufacturer's instructions of the ECL kit. The exposed X-ray film was scanned using Tanon Image Note (Tanon Science \& Technology Co., Ltd., Shanghai, China) for grayscale analysis, and $\beta$-actin was adopted as an internal reference.

MTT assay of cell growth. The present study included five experimental groups, which were termed the RNAi group, the RAPA group (culture medium containing $9.14 \mathrm{mg} / \mathrm{l}$ RAPA) (18), the RNAi + RAPA group (pU6mRFP RhoC-siRNA transfected $24 \mathrm{~h}$ after RAPA administration), the Scramble group (transfected with pU6m RFPscramble-siRNA) and the HL7702 normal hepatocyte cell (Shanghai Institute of Biochemistry and Cell Biology Cell Bank) group. A total of $2 \times 10^{3}$ cells/well were seeded into a 96-well plate, with a total reaction system at $200 \mu \mathrm{l}$ per well and incubated for seven days. MTT (5 mg/ml; $10 \mu \mathrm{l}$; Sigma-Aldrich) was added to each well, then the culture was continued for $6 \mathrm{~h}$ prior to the detection. The culture medium was discarded, dimethyl sulfoxide (Sigma-Aldrich) was added (100 $\mu \mathrm{l} /$ well) and then the solution was mixed with vortexing for $5 \mathrm{~min}$. The absorbance was measured by a microplate reader (ELx800; Bio-Tek Instruments, Inc., Winooski, VT, USA) at $490 \mathrm{~nm}$ wavelength. The experiment was performed in triplicate for each group.

Argyrophilic protein analysis of cell proliferation. Cells from each group were seeded in 24-well culture plates containing cover slips. The culture plates were placed in an incubator at $37^{\circ} \mathrm{C}$, with $5 \% \mathrm{CO}_{2}$. When cultivated cells grew to a monolayer, the cover slips were removed and cells were fixed in 95\% ethanol. The samples were immersed in deionized water for hydration; then, $\sim 5$ drops ( $20 \mu \mathrm{l} / \mathrm{drop}$ ) of silver nitrate solution ( $0.2 \mathrm{~g}$ gelatin, $10 \mathrm{ml} \mathrm{1 \%}$ formic acid, $20 \mathrm{ml} 50 \%$ silver nitrate; Sigma-Aldrich) were added onto the cover slips under the exclusion of light, followed by incubation at room temperature for $1 \mathrm{~h}$. The cells were repeatedly washed with deionized water, dehydrated with $95-100 \%$ alcohol, cleared with xylene and mounted with neutral gum (Sigma-Aldrich). Single black particles in the nucleus were counted under a light microscope (CKX41; Olympus, Tokyo, Japan) and three horizons were randomly counted.

Wright's stain to detect cell apoptosis. Cells in the logarithmic growth phase were harvested, with $\sim 1 \times 10^{6}$ cells in each group, and then washed with PBS. Cell were adjusted to $1 \times 10^{5}$ cells $/ \mathrm{ml}$, inoculated in a 12-well culture plate $(1 \mathrm{ml} /$ well), with a cover slip at the bottom of each well. A total of $24 \mathrm{~h}$ subsequent to incubation, each cover slip was washed carefully with $3 \mathrm{ml}$ PBS three times. The cell slides were then fixed with methanol for 3-5 min, stained with Wright's solution (Hartman-Leddon Company, Inc., Philadelphia, PA, USA) for $2 \mathrm{~min}$, immersed into Wright's phosphate buffer (Wright's solution:PBS, 1:2) dilution for 4-10 min and then rinsed with distilled water. The cell slides were mounted with neutral gum after open-air drying. Images were captured under a microscope.

Soft agar colony formation assays. Cells in the logarithmic growth phase were adjusted to a density of $1 \times 10^{3} / \mathrm{ml}$. Agar (5\%; Sigma-Aldrich) was completely dissolved in a boiling water bath. A portion of the $5 \%$ agar was transferred into a small beaker, cooled to $50^{\circ} \mathrm{C}$ and rapidly mixed with nine portions of fresh pre-warmed culture medium at $37^{\circ} \mathrm{C}$. The mixture was immediately poured into 24 -well culture plates, with each well containing $0.8 \mathrm{ml} 0.5 \%$ agar medium and then solidified at room temperature. A $0.3 \%$ agar medium was prepared from $9.4 \mathrm{ml}$ cell suspension in a small beaker (incubated at $37^{\circ} \mathrm{C}$ ) by rapid mixing with $0.6 \mathrm{ml} 5 \%$ agar at $50^{\circ} \mathrm{C}$. The agar culture was immediately poured into 24-well plates, which were covered with agar at their base, with $0.8 \mathrm{ml}$ medium in each well, and solidified at room temperature. A total of 500 cells were added per well. The plates were placed into an incubator at $37^{\circ} \mathrm{C}$ with $5 \% \mathrm{CO}_{2}$ and saturated humidity for two weeks. The clone forming efficiency was counted when cell clones were $>75 \mu \mathrm{m}$ in diameter or $>50$ cells in a single clone were observed under an inverted microscope. The following equation was used to calculate the clone formation percentage: Clone formation percentage $=$ number of clones - forming/seeded cells $\times 100 \%$.

Transwell assay for detection of cell migration. Cells in the logarithmic growth phase were digested with trypsin (Amresco LLC, Solon OH, USA) to prepare a cell suspension of $4 \times 10^{6}$ cells $/ \mathrm{ml}$. A total of $200 \mu \mathrm{l}$ cell suspension was added into the upper chambers of a Transwell plate (Shanghai Yu Bo Biological Technology Co., Ltd., Shanghai, China), and $500 \mu \mathrm{l}$ culture medium containing penicillin and streptomycin was added into the lower chamber, followed by incubation at $37^{\circ} \mathrm{C}$ for $24 \mathrm{~h}$. The culture medium was discarded, and non-migrating cells were collected with a cotton swab. Cells were washed twice with PBS, fixed with 4\% paraformaldehyde for $20 \mathrm{~min}$ then flushed with distilled water. A total of $500 \mu 10.1 \%$ crystal violet was added into the chamber, incubated at $37^{\circ} \mathrm{C}$ for $30 \mathrm{~min}$ and then washed with distilled water. The microscope was used to observe migrated cells. Three visual fields were randomly selected for counting and capturing of images. 
A

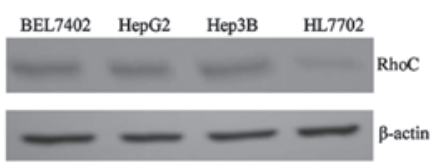

B

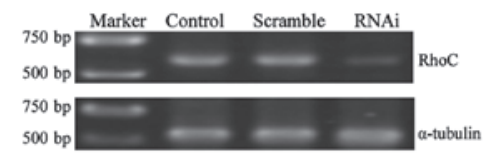

C

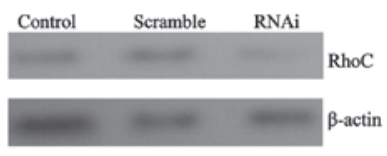

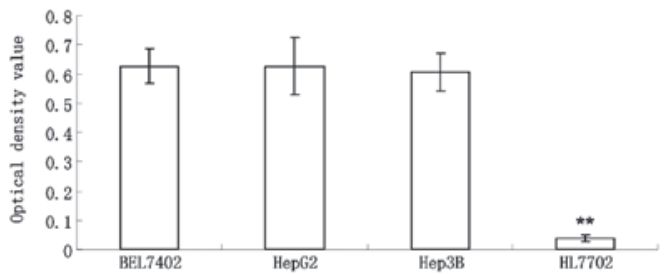
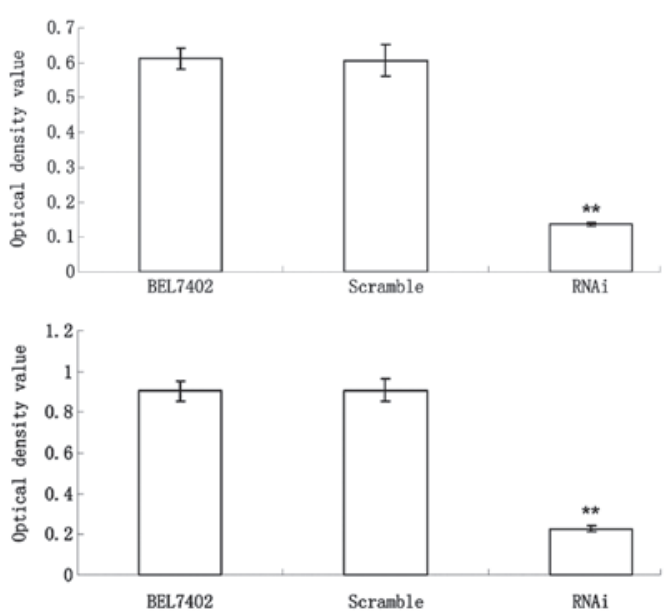

Figure 1. RhoC expression in hepatoma cell lines and a normal liver cell line and RhoC knockdown. (A) Western blot analysis of RhoC. RhoC protein expression in HL7702 normal liver cells was lower than that in hepatoma cells. (B) Semi-quantitative polymerase chain reaction analysis of RhoC mRNA levels in BEL7402 cells following RhoC knockdown. RhoC mRNA expression was markedly decreased by RhoC-siRNA. (C) Western blot analysis of RhoC in BEL7402 cells following RhoC knockdown. Values are expressed as the mean \pm standard deviation, ${ }^{* *} \mathrm{P}<0.01$. RhoC, Ras homolog gene family, member $\mathrm{C}$; siRNA, small interfering RNA; RNAi, RNA interference.

Statistical analysis. Values are expressed as the mean \pm standard deviation. Experiments were repeated three times. SPSS 13.0 software (SPSS, Inc., Chicago, IL, USA) was used for statistical analysis, and the t-test as well as an analysis of variance were performed. $\mathrm{P}<0.05$ was considered to indicate a statistically significant difference.

\section{Results}

RhoC gene expression and RNAi gene silencing effects. RhoC expression in the HL7702 normal human liver cell line was significantly lower than that in BEL7402, HepG2 and Hep3B hepatoma cells $(0.04 \pm 0.01$ vs. $0.63 \pm 0.06,0.63 \pm 0.10$ and $0.61 \pm 0.07$; $\mathrm{P}<0.01$ ) (Fig. $1 \mathrm{~A}$ ). RhoC mRNA expression in the RNAi group decreased significantly compared with that in the control and Scramble groups $(0.26 \pm 0.02$ vs. $0.90 \pm 0.05$ and $0.91 \pm 0.06 ; \mathrm{P}<0.01$ ). The inhibition efficiency was $\sim 75 \%$ (Fig. 1B), suggesting that RhoC-siRNA had a good gene silencing effect. Western blot analysis revealed that RhoC protein expression in the RNAi group was significantly lower than that in the control and scramble group, with an inhibition ratio of $78.2 \%(\mathrm{P}<0.01$, Fig. $1 \mathrm{C})$, in accordance with the PCR results.

Combined RhoC knockdown and RAPA treatment decrease the proliferation of hepatoma cells. From the 2nd day of RAPA treatment, the rate of cell proliferation in the RNAi + RAPA group was significantly lower than that in the RNAi, RAPA and Scramble groups $(0.27 \pm 0.02$ vs. $0.34 \pm 0.02$, $0.37 \pm 0.02,0.41 \pm 0.02 ; \mathrm{P}<0.05$ ), with no significant difference compared with that in the normal HL7702 group $(0.27 \pm 0.02$ vs. $0.26 \pm 0.03 ; \mathrm{P}>0.05$ ) (Fig. 2A). Cell proliferation-associated gene expression revealed that cyclin-dependent kinase 2 (CDK2) expression in the RNAi + RAPA group was significantly lower than that in the RNAi, RAPA and Scramble groups $(0.10 \pm 0.02$ vs. $0.52 \pm 0.03,0.54 \pm 0.02$ and $0.81 \pm 0.03$; $\mathrm{P}<0.01)$. No significant difference was observed between the RNAi + RAPA and HL7702 groups $(0.10 \pm 0.02$ vs. $0.07 \pm 0.01$; $\mathrm{P}>0.05$ ) (Fig. 2B). P16 gene expression in the RNAi + RAPA group was significantly higher than that in the RNAi, RAPA and Scramble groups $(0.72 \pm 0.04$ vs. $0.27 \pm 0.04,0.29 \pm 0.04$ and $0.09 \pm 0.03 ; \mathrm{P}<0.01)$, and no significant difference was found when compared with the HL7702 group $(0.72 \pm 0.04$ vs. $0.77 \pm 0.05 ; \mathrm{P}>0.05$ ) (Fig. 2B).

In the silver nitrate staining assay, the number of stained particles in the nuclei in the RNAi + RAPA group was significantly reduced when compared with that in the RNAi, RAPA and Scramble groups $(1.22 \pm 0.44$ vs. $2.22 \pm 0.44,2.44 \pm 0.53$ and $3.78 \pm 0.67$; P $<0.01$ ) (Fig. 3). No notable difference was found between the RNAi + RAPA and HL7702 groups $(1.22 \pm 0.44$ vs. $1.33 \pm 0.50 ; \mathrm{P}>0.05)$. Only a small number of stained particles were observed in the RNAi + RAPA group, indicating decreased cell proliferation, which was consistent with the results of the MTT assay.

All of these results suggested that RhoC knockdown and RAPA treatment had synergic effects in inhibiting hematoma cell proliferation.

RhoC knockdown and RAPA treatment increase the apoptotic rate and apoptosis-associated signaling in hepatoma cells. Wright's staining revealed that the cells in the Scramble group were homogeneous and that apoptosis was scarce. 


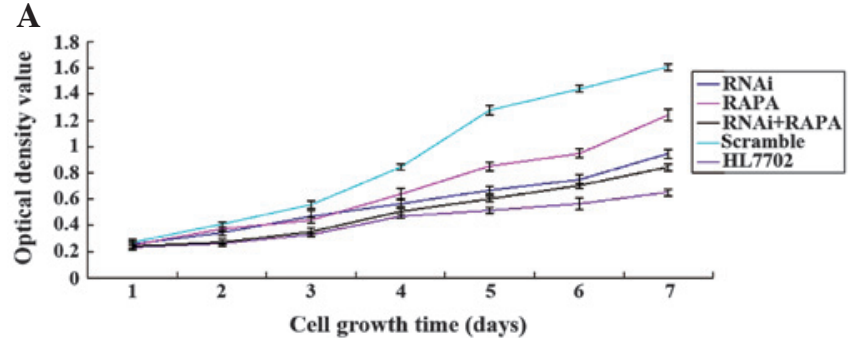

B
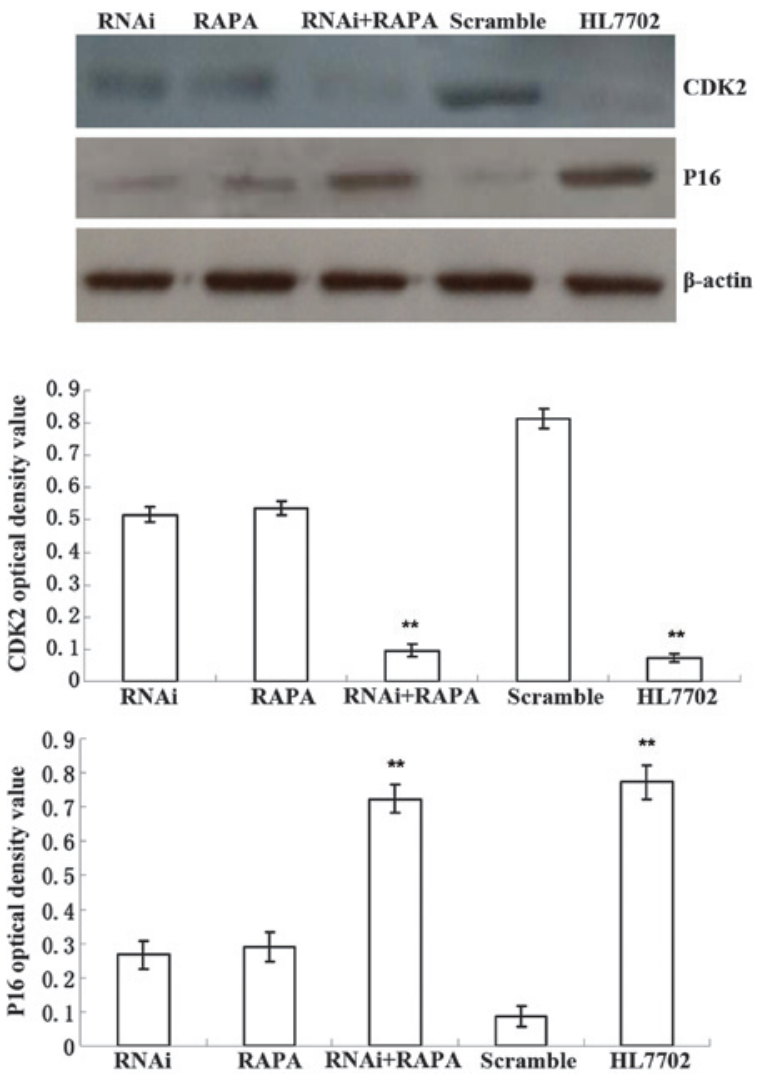

Figure 2. Cell proliferation and expression of cell cycle-associated genes. (A) Cell proliferation in the RNAi + RAPA and HL7702 group was markedly suppressed compared with that in the other groups. (B) Western blot analysis showed that CDK2 expression was decreased in the RNAi + RAPA group, while P16 was greatly increased in the RNAi + RAPA. Values are expressed as the mean \pm standard deviation, ${ }^{* *} \mathrm{P}<0.01$. RAPA, rapamycin; RNAi, RNA interference; $\mathrm{CDK}$, cyclin-dependent kinase.

The RNAi + RAPA, RNAi and RAPA groups contained a large number of apoptotic cells, which displayed nuclear condensation, fragmentation, deepened chromatin staining and wrinkled membranes, with the apoptotic rate in the RNAi + RAPA group being higher than that in the other experimental groups. The HL7702 cells also exhibited a significant level of apoptosis. The examination of the expression of apoptosis-associated genes demonstrated that B-cell lymphoma-2 (Bcl-2) expression in the RNAi + RAPA group was significantly lower than that in the RNAi, RAPA and Scramble groups $(0.09 \pm 0.02$ vs. $0.32 \pm 0.05,0.34 \pm 0.05$ and $0.60 \pm 0.04 ; \mathrm{P}<0.01)$, with no significant difference compared with that in the HL7702 group $(0.09 \pm 0.02$ vs. $0.08 \pm 0.03$; $\mathrm{P}>0.05$ ) (Fig. 4). Bcl-2-associated X protein (Bax) gene expression in the RNAi + RAPA group was significantly higher than that in the RNAi, RAPA and Scramble groups
A
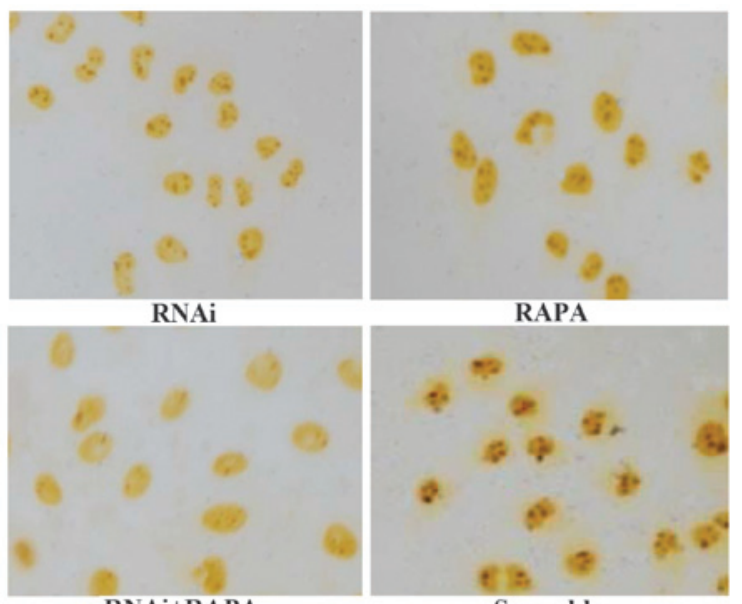

RNAi+RAPA
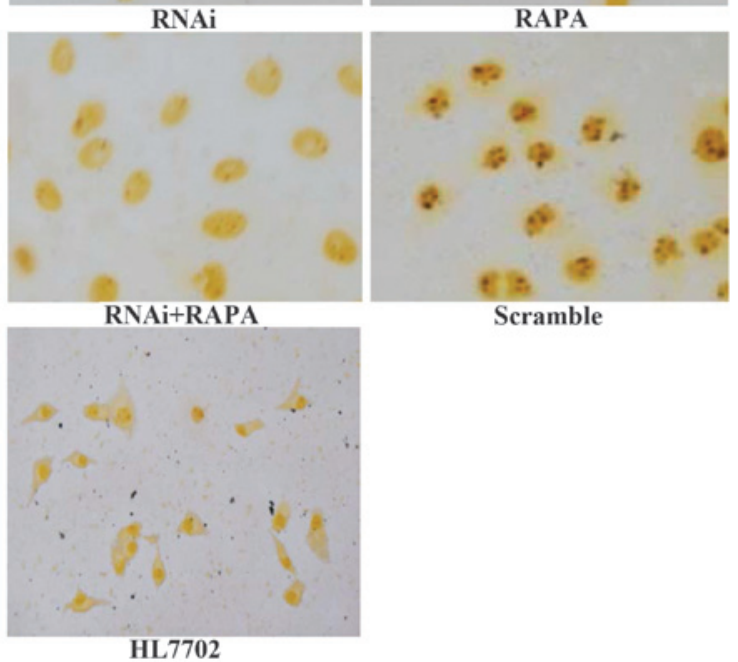

B
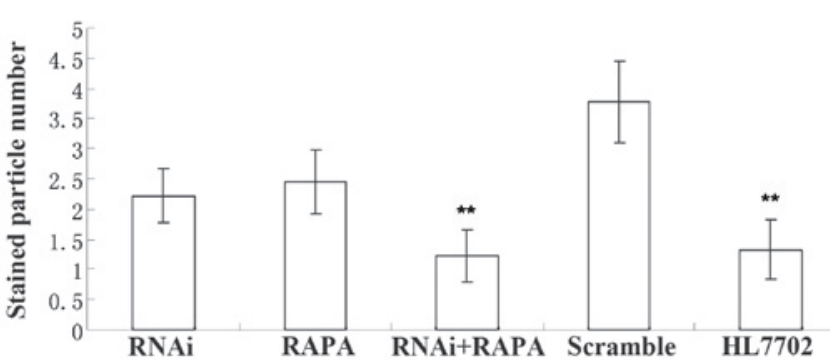

Figure 3. Cells stained with silver nitrate in each experimental group. Silver nitrate-stained particles in the RNAi + RAPA and HL7702 groups were rare. (A) Representative microscopic images (magnification, x200). Brown particles indicate proliferation-associated silver nitrate staining. (B) Quantified results demonstrate that the number of silver-stained particles in the RNAi + RAPA group was decreased to the level of the HL7702 group. Values are expressed as the mean \pm standard deviation, ${ }^{* *} \mathrm{P}<0.01$. RAPA, rapamycin; RNAi, RNA interference.

$(0.64 \pm 0.08$ vs. $0.31 \pm 0.04,0.33 \pm 0.04$ and $0.27 \pm 0.04 ; \mathrm{P}<0.01)$. There was no significant difference in Bax or Bcl-2 levels between the RNAi + RAPA and HL7702 groups $(0.64 \pm 0.08$ vs. $0.53 \pm 0.07 ; \mathrm{P}>0.05$ ) (Fig. 4).

RhoC knockdown and RAPA treatment inhibit cell colony formation of hepatoma cells. The soft agar assay showed that cell colony-formation in the RNAi and RAPA groups was scarce, while no colony formation was observed in the RNAi + RAPA and HL7702 groups (Fig. 5). Soft agar cell colony formation in the RNAi group was significantly lower than that in the Scramble group $(1.67 \pm 0.58$ vs. $12.26 \pm 2.84$; $\mathrm{P}<0.01)$, and the difference between the RNAi and RAPA groups was not significant $(1.67 \pm 0.58$ vs. $3.33 \pm 1.53 ; \mathrm{P}>0.05)$ (Fig. 5).

RhoC knockdown and RAPA treatment decrease cell migration as well as migration-associated protein expression in hepatoma cells. The Transwell assay showed that cells 


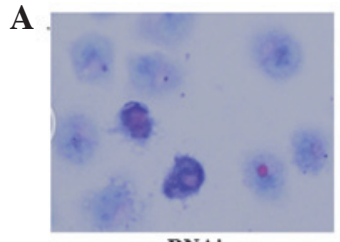

RNAi

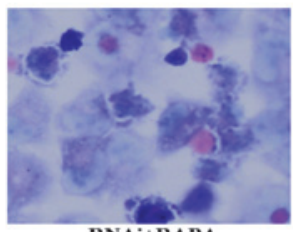

RNAi+RAPA

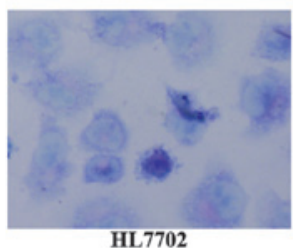

B
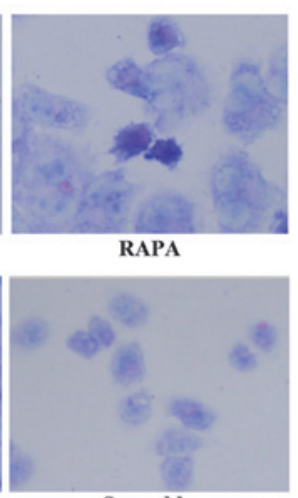

Scramble
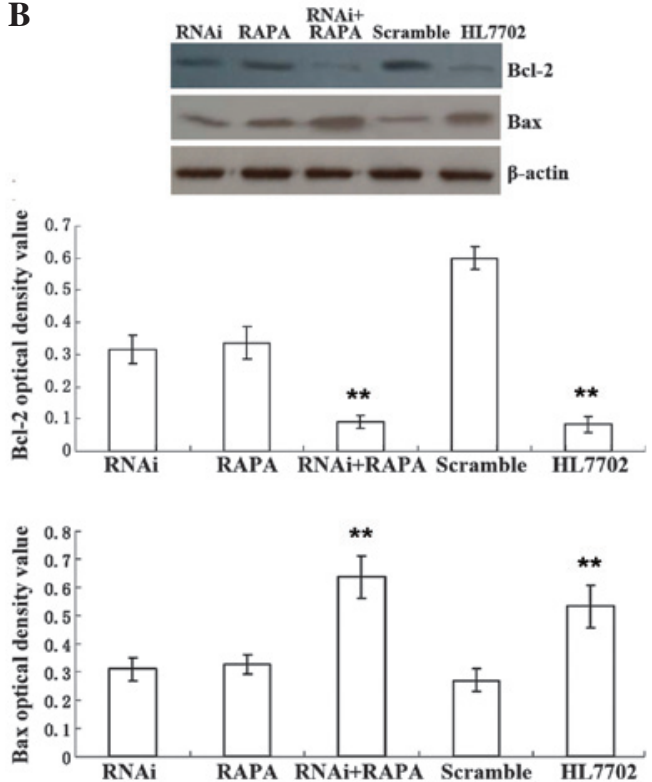

Figure 4. Apoptosis and expression of apoptosis-associated genes. (A) Microscopic images (magnification, x200). The number of apoptotic cells in the RNAi + RAPA group was greater than that in the other groups. (B) Western blot analysis of Bcl-2 and Bax. Bcl-2 expression in the RNAi + RAPA group was greatly decreased to the level of the HL7702 group, while Bax expression was largely increased to the level of the HL7702 group. Values are expressed as the mean \pm standard deviation, ${ }^{* *} \mathrm{P}<0.01$. RAPA, rapamycin; RNAi, RNA interference; Bax, Bcl-2-associated X protein; Bcl-2, B cell lymphoma-2.

A
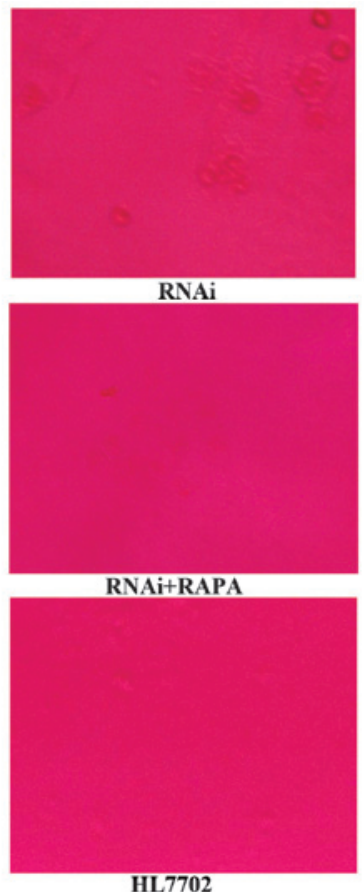

B
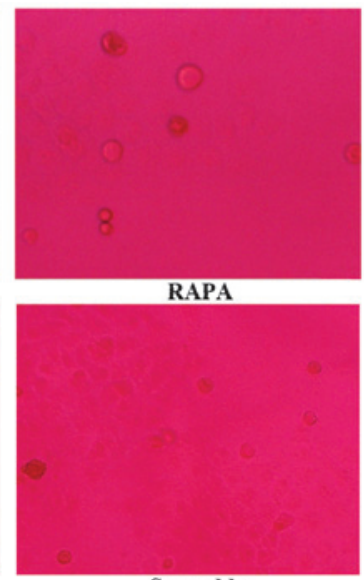

Scramble

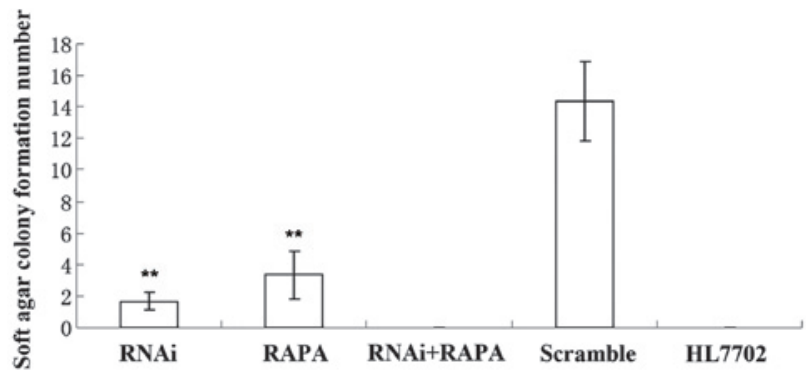

Figure 5. Soft agar colony formation in different cell groups.(A) Representative microscopic images (magnification, $\mathrm{x} 40$ ); (B) Statistical evaluation of A. Values are expressed as the mean \pm standard deviation, ${ }^{* *} \mathrm{P}<0.01$. RAPA, rapamycin; RNAi, RNA interference. which migrated across the membrane in the RNAi, RAPA, RNAi + RAPA and HL7702 groups were rare and scattered, while in the Scramble group, transmembrane cells were greater in number and dense in distribution. Statistical analysis revealed that the number of transmembrane cells in the RNAi + RAPA group was significantly lower than that in the RNAi, RAPA and Scramble groups ( $35.33 \pm 3.51$ vs. $65.33 \pm 6.51$, $60.67 \pm 8.50$ and $161.67 \pm 14.50 ; \mathrm{P}<0.01)$, and no significant difference was found when compared with the HL7702 group (35.33 \pm 3.51 vs. $25.67 \pm 5.86$; $\mathrm{P}>0.05$ ) (Fig. 6 A). Detection of cell migration-associated genes revealed that matrix metalloproteinase (MMP)-2 expression in the RNAi + RAPA group was significantly lower than that in the RNAi, RAPA and Scramble groups $(0.04 \pm 0.02$ vs. $0.21 \pm 0.03,0.20 \pm 0.04$ and $0.67 \pm 0.04 ; \mathrm{P}<0.01$ ), and the difference was not significant compared with that in the HL7702 group $(0.04 \pm 0.02$ vs. $0.03 \pm 0.01 ; \mathrm{P}>0.05$ ). MMP-9 expression in the RNAi + RAPA group was markedly decreased compared with that in the RNAi, RAPA and Scramble groups $(0.06 \pm 0.02$ vs. $0.23 \pm 0.02$, $0.19 \pm 0.04$ and $0.62 \pm 0.03 ; \mathrm{P}<.0 .01$ ), and no significant difference was observed when compared with that in the HL7702 group $(0.06 \pm 0.02$ vs. $0.03 \pm 0.01 ; \mathrm{P}>0.05)$. VEGF expression in the RNAi + RAPA group was also significantly lower than that in the RNAi, RAPA and Scramble groups $(0.07 \pm 0.02$ vs. $0.24 \pm 0.04,0.20 \pm 0.06$ and $0.62 \pm 0.06 ; \mathrm{P}<0.01$ ); while there was no significant difference compared with that in the HL7702 group $(0.07 \pm 0.02$ vs. $0.04 \pm 0.02 ; \mathrm{P}>0.05)$ (Fig. $6 \mathrm{~B})$. These results demonstrated that $\mathrm{RhoC}$ gene silencing combined with RAPA treatment exerted marked migration-inhibitory effects on hepatocellular carcinoma cells.

\section{Discussion}

Liver cancer results from the imbalance of cell proliferation and apoptosis, involving complex biological processes 
A
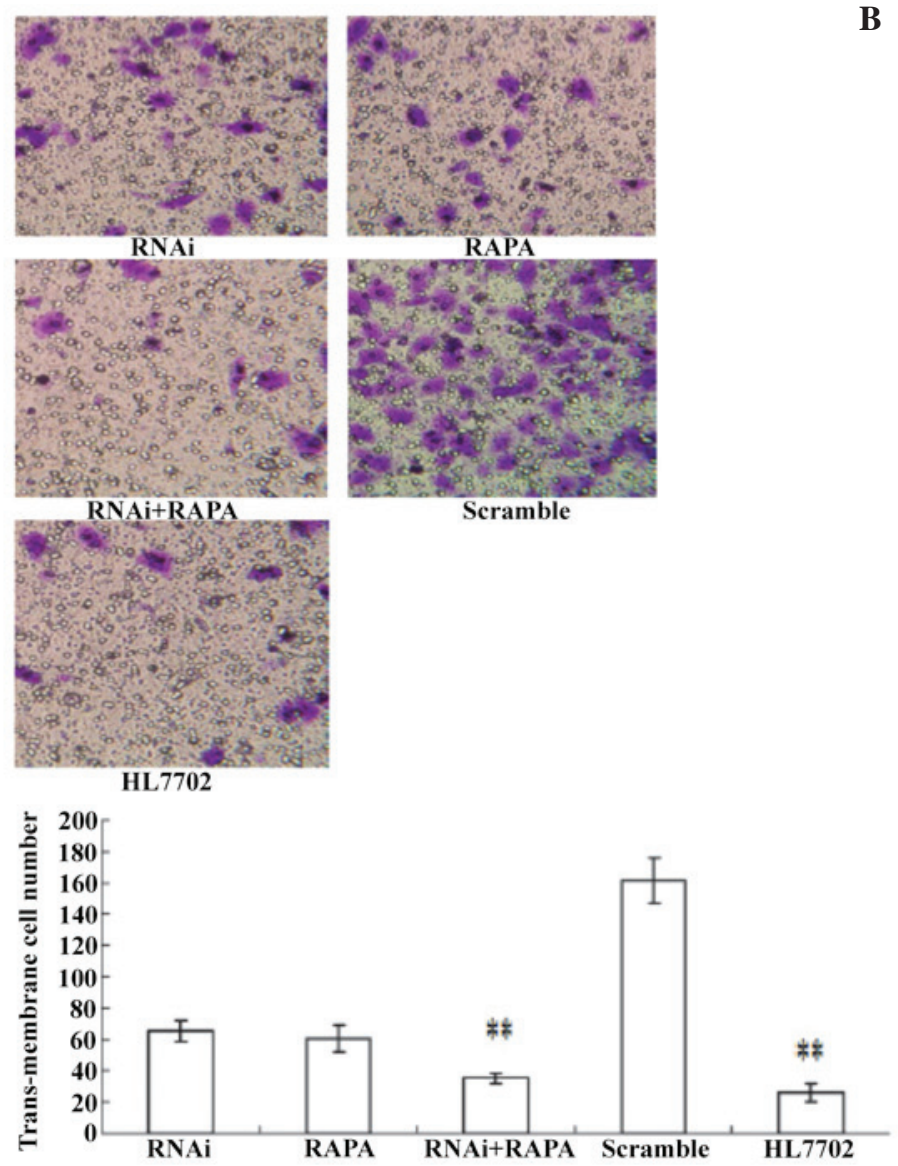

B

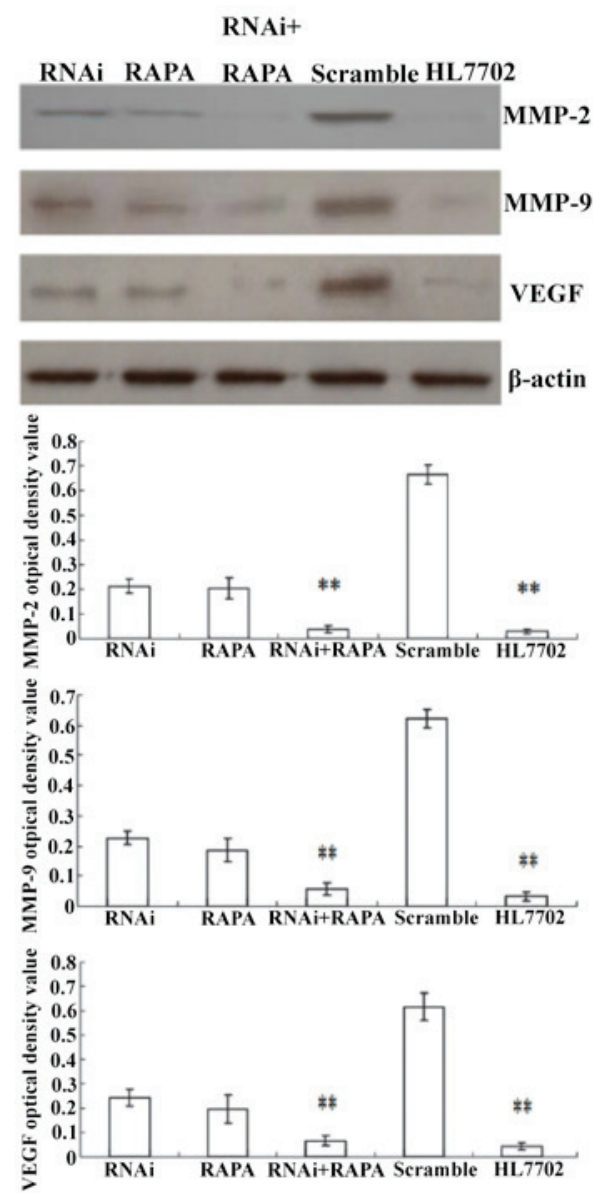

Figure 6. Cell migration and expression of migration-associated genes. (A) Representative microscopic images (magnification, x100) and statistical evaluation of migrated cells. Transmembrane cells in the RNAi + RAPA and HL7702 groups were rare and sparsely distributed, while in the Scramble group were greater in quantity and widely distributed. (B) Western blot analysis of migration-associated proteins. MMP2/9 and VEGF protein expression were decreased in the RNAi + RAPA group to the levels of HL7702. Values are expressed as the mean \pm standard deviation, ${ }^{* *} \mathrm{P}<0.01$. RAPA, rapamycin; RNAi, RNA interference; MMP, matrix metalloproteinase; VEGF, vascular endothelial growth factor.

in which cell signaling pathways are important (19). To examine the combined effects of inhibiting the RhoC/RhoC kinase (ROCK) and PI3K/Akt/mTOR pathways on hepatocellular carcinoma cell growth, RhoC gene silencing and RAPA treatment were applied. It was identified that inhibition of the two pathways had synergistic effects and more effectively inhibited the proliferation, metastasis and apoptosis of hepatoma cells as compared with monotreatment.

RhoC, as a Rho subfamily member, is an important intracellular signal transduction molecule. Extracellular stimulus signals are able to promote the Rho guanosine diphosphate-bound form of the protein from the inactive state into the active guanosine triphosphate-bound state, affecting the cell biological character by regulating the metabolism of tumor cell growth, invasion and migration (20). RhoC regulates the cytoskeleton through actin stress fibers and focal adhesions, thereby affecting cell migration, invasion and other functions. In a number of cancer types, including colorectal, liver, breast and prostate cancer, abnormal expression of RhoC is necessary, and is important in tumor growth, invasion and metastasis (21). The present study identified RhoC overexpression in three types of hepatoma cell. By contrast, RhoC expression was low in normal liver cells, consistent with the literature (19). A previous study by our group reported that RhoC gene silencing was able to inhibit tumor cell growth, migration and invasion, and promoted tumor cell apoptosis (7).

The PI3K/AKT/mTOR signaling pathway has been widely elucidated. It is involved in intracellular signal transduction of several growth factors, and is closely associated with the biological function of certain diseases (13). mTOR is the core molecule of this pathway, and its sustained activation is associated with a variety of tumor behaviors, including invasion and metastasis, tumor recurrence, and patient prognosis (9). mTOR consists of two distinct multiprotein complexes: mTORC1 and mTORC2. RAPA is sensitive to mTORC1 and can markedly inhibit its activities. mTORC1 activates S6K1 and 4EBP1, which are associated with mRNA translation. Diverse stimuli can activate mTORC1, such as growth factors, nutrients, energy and stress signals, and essential signaling pathways, such as PI3K, mitogen-activated protein kinases and AMP-activated protein kinase, in order to control cell growth, proliferation and survival (22). The RhoC gene silencing experiments in combination with RAPA administration achieved greater effects than monotreatment. As RAPA is an mTOR inhibitor, it is able to inhibit the PI3K/Akt $/ \mathrm{mTOR}$ pathway, has a marked cytostatic effect, and, in combination with RhoC gene silencing, produced a synergistic effect, 
markedly inhibiting liver cancer cell proliferation and migration, as well as promoting apoptosis.

Normal operation of the cell cycle requires the joint action of CDKs and cyclins. Certain CDKs and cyclins are also molecular targets for cancer therapy. Studies have attempted to interfere with these regulatory molecules for the treatment of tumors (23). To examine the mechanism of cell growth inhibition, the present study selected two cell cycle-associated molecules to investigate. One was CDK2, which promotes cell growth, and the other was P16, which inhibits cell growth. The results revealed that RhoC gene silencing combined with RAPA administration markedly decreased CDK2 expression and greatly increased P16 expression. The observed changes in these two important cell cycle regulatory molecules are known to hamper cell cycle progression between the G1 and S phase, thereby inhibiting cell proliferation. Silver nitrate staining confirmed the improved inhibitory effect of the combined efforts. In addition, the combined effects also changed the characteristics of liver cancer growth, rendering hepatocellular carcinoma cells unable to grow in soft agar.

Local spread and distant metastasis of cancer cells is the major cause of mortality in patients with hepatocellular carcinoma. In the process of tumor invasion and metastasis, the extracellular basement membrane is an important target, other than tumor angiogenesis. Tumor invasion of the basement membrane can be divided into three steps. Firstly, cell adhesion to the basement membrane; secondly, extracellular matrix (ECM) degradation, and finally the migration of cells. The ECM is a complex structure, composed of a variety of constituents, predominantly type IV collagen, fibronectin and laminin, amongst others. It is a natural barrier for tumor cell invasion and metastasis (24). Tumor cell invasion and metastasis must penetrate the barrier of the ECM. The Transwell chamber experiment is able to simulate the process of tumor cell invasion. Aggressive cells adhere, deform and move through the bottom of the filter chamber, demonstrating the ability of tumor cells to invade and migrate through the basement membrane. RAPA combined with RhoC gene silencing decreased the capacity of tumor cells to pass through the Transwell membrane, indicating a reduced invasiveness of the hepatoma cells.

Damage to the ECM barrier is the most important process in tumor cell invasion and metastasis. Tumor cells are activated by adhering to the matrix through cell surface receptors, and secreting various proteolytic enzymes. Matrix metalloproteinases (MMPs) are the main connective tissue-degrading enzymes. ECM and the main structural protein of the basement membrane are composed of type IV collagen, and MMP-2 and MMP-9 are the most important enzymes for type IV collagen degradation, involving the whole process of basement membrane degradation. A previous study found that MMP-7, MMP-9 and tissue inhibitor of metalloproteinase expression in non-small cell lung cancer were significantly higher than that in the surrounding tissues (24). The activated PI3K/Akt/mTOR signaling pathway may also upregulate MMP gene expression (25). It was hypothesized that RhoC gene silencing combined with RAPA administration may decrease MMP expression and activity, and suppress ECM degradation and tumor cell migration, thus preventing tumor cell invasion and metastasis.

VEGF is a vascular endothelial-specific mitogen, regulating vascular endothelial cell proliferation and migration. Although it stimulates tumor angiogenesis, it increases vascular permeability and plasma protein extravasation, providing a matrix for cancer metastasis. MMP-9 promotes the migration of vascular endothelial cells, involving the process of tumor neovascularization through the degradation of the basement membrane and the ECM. Following specific binding with its receptor, VEGF is able to facilitate vascular cell proliferation, increasing vascular permeability and changing endothelial cell gene expression, and thereby enhancing MMP synthesis (26). According to the present experimental results, it is reasonable to hypothesize that $\mathrm{RhoC}$ gene silencing and RAPA administration are able to suppress the VEGF-induced tumor vasculature, thereby inhibiting liver cancer cell invasion and metastasis, and their combination can further enhance this inhibitory effect.

An important cause of the fatalities in patients with liver cancer is chemical therapy resistance, which is closely associated with a loss of balance of Bcl-2/Bax genes (27). RAPA administration combined with RhoC gene silencing inhibited $\mathrm{Bcl}-2$ expression and promoted Bax expression. $\mathrm{Bcl}-2$ is one of the most well known apoptosis-associated genes, which is closely linked with cancer. The Bcl-2 family consists of two groups of genes with antagonistic functions. Bcl-2, Bcl-like protein 2 and $\mathrm{Bcl}$ extra large suppress apoptosis, while Bax, $\mathrm{Bcl}$-2-associated death promoter and $\mathrm{BH} 3$ interacting-domain death agonist promote apoptosis. Bcl-2 affects tumor development by inhibiting cell apoptosis and prolonging cell survival. The ratio of intracellular Bcl-2/Bax regulates cell apoptosis. Bax homodimer formation can induce cell apoptosis. With elevated Bcl-2 expression, Bax homodimer separates, combining with $\mathrm{Bcl}-2$ to form a Bax-Bcl-2 heterodimer, which is more stable than the Bax-Bax homodimer, thereby offsetting the Bax-induced apoptosis. Wright's staining morphologically confirmed that RAPA administration combined with RhoC silencing had enhanced pro-apoptotic effects on hepatoma cells.

In conclusion, the present study demonstrated that silencing of RhoC combined with RAPA administration was able to inhibit the rapid proliferation of hepatocellular carcinoma cells, and deter tumor cell invasion and metastasis. Its mechanism may be associated with the synergistic effects of inhibiting the Rho/ROCK and PI3K/Akt/mTOR signaling pathways in liver cancer cells.

\section{References}

1. Abdel-Rahman O: Systemic therapy for hepatocellular carcinoma (HCC): From bench to bedside. J Egypt Natl Canc Inst 25: 165-171, 2013.

2. Harrold JM, Ramanathan M and Mager DE: Network-based approaches in drug discovery and early development. Clin Pharmacol Ther 94: 651-658, 2013.

3. Zhao Y, Zheng HC, Chen S, Gou WF, Xiao LJ and Niu ZF: The role of RhoC in ovarian epithelial carcinoma: A marker for carcinogenesis, progression, prognosis and target therapy. Gynecol Oncol 130: 570-578, 2013.

4. Islam M, Sharma S, Kumar B and Teknos TN: Atorvastatin inhibits RhoC function and limits head and neck cancer metastasis. Oral Oncol 49: 778-786, 2013.

5. Wu Y, Chen YC, Sang JR and Xu WR: RhoC protein stimulates migration of gastric cancer cells through interaction with scaffold protein IQGAP1. Mol Med Rep 4: 697-703, 2011.

6. Wang W, Yang LY, Huang GW, Lu WQ, Yang ZL, Yang JQ and Liu HL: Genomic analysis reveals RhoC as a potential marker in hepatocellular carcinoma with poor prognosis. Br J Cancer 90: 2349-2355, 2004. 
7. Xie S, Zhu M, Lv G, Zhang Q and Wang G: The role of RhoC in the proliferation and apoptosis of hepatocellular carcinoma cells. Med Oncol 29: 1802-1809, 2012.

8. Xie S, Zhu M, Lv G, Geng Y, Chen G, Ma J and Wang G: Overexpression of Ras homologous C (RhoC) induces malignant transformation of hepatocytes in vitro and in nude mouse xenografts. PLoS One 8: e54493, 2013.

9. Psyrri A, Arkadopoulos N, Vassilakopoulou M, Smyrniotis V and Dimitriadis G: Pathways and targets in hepatocellular carcinoma. Expert Rev Anticancer Ther 12: 1347-1357, 2012.

10. Yang H, Rudge DG, Koos JD, Vaidialingam B, Yang HJ and Pavletich NP: mTOR kinase structure, mechanism and regulation. Nature 497: 217-223, 2013.

11. Diaz-Padilla I, Duran I, Clarke BA and Oza AM: Biologic rationale and clinical activity of mTOR inhibitors in gynecological cancer. Cancer Treat Rev 38: 767-775, 2012.

12. Sendur MA, Aksoy S, Zengin N and Altundag K: Comparative efficacy study of 5-year letrozole or anastrozole in postmenopausal hormone receptor-positive early breast cancer. J BUON 18 838-844, 2013.

13. Khan KH, Yap TA, Yan L and Cunningham D: Targeting the PI3K-AKT-mTOR signaling network in cancer. Chin J Cancer 32: 253-265, 2013

14. Yamanaka K, Petrulionis M, Lin S, Gao C, Galli U, Richter S, Winkler S, Houben P, Schultze D, Hatano E, et al: Therapeutic potential and adverse events of everolimus for treatment of hepatocellular carcinoma-systematic review and meta-analysis. Cancer Med 2: 862-871, 2013.

15. Finn RS: Current and future treatment strategies for patients with advanced hepatocellular carcinoma: Role of mtor inhibition. Liver Cancer 1: 247-256, 2012.

16. Ruth MC, Xu Y, Maxwell IH, Ahn NG, Norris DA and Shellman YG: RhoC promotes human melanoma invasion in a PI3K/Akt-dependent pathway. J Invest Dermatol 126: 862-868, 2006.
17. Lehman HL, Van Laere SJ, van Golen CM, Vermeulen PB, Dirix LY and van Golen KL: Regulation of inflammatory breast cancer cell invasion through Akt1/PKB $\alpha$ phosphorylation of RhoC GTPase. Mol Cancer Res 10: 1306-1318, 2012.

18. Jin Z: Experimental research on the combined effects of RhoC-siRNA and rapamycin in hepatocellular carcinoma cell line. Master Thesis of Jilin University 34-36, 2010.

19. Grise F, Bidaud A and Moreau V: Rho GTPases in hepatocellular carcinoma. Biochim Biophys Acta 1795: 137-151, 2009.

20. Wilson KF, Erickson JW, Antonyak MA and Cerione RA: Rho GTPases and their roles in cancer metabolism. Trends Mol Med 19: 74-82, 2013.

21. Karlsson R, Pedersen ED, Wang Z and Brakebusch C: Rho GTPase function in tumorigenesis. Biochim Biophys Acta 1796: 91-98, 2009.

22. Populo H, Lopes JM and Soares P: The mTOR signalling pathway in human cancer. Int J Mol Sci 13: 1886-1918, 2012.

23. Diaz-Moralli S, Tarrado-Castellarnau M, Miranda A and Cascante M: Targeting cell cycle regulation in cancer therapy. Pharmacol Ther 138: 255-271, 2013.

24. Safranek J, Pesta M, Holubec L, Kulda V, Dreslerova J, Vrzalova J, Topolcan O, Pesek M, Finek J and Treska V: Expression of MMP-7, MMP-9, TIMP-1 and TIMP-2 mRNA in lung tissue of patients with non-small cell lung cancer (NSCLC) and benign pulmonary disease. Anticancer Res 29: 2513-2517, 2009.

25. Wu MH, Lo JF, Kuo CH, Lin JA, Lin YM, Chen LM, Tsai FJ, Tsai $\mathrm{CH}$, Huang $\mathrm{CY}$ and Tang $\mathrm{CH}$ : Endothelin-1 promotes MMP-13 production and migration in human chondrosarcoma cells through FAK/PI3K/Akt/mTOR pathways. J Cell Physiol 227: 3016-3026, 2012.

26. Chaudhary AK, Pandya S, Ghosh K and Nadkarni A: Matrix metalloproteinase and its drug targets therapy in solid and hematological malignancies: An overview. Mutat Res 753: 7-23, 2013.

27. Liu Z, Cheng M and Cao M: Potential targets for molecular imaging of apoptosis resistance in hepatocellular carcinoma. Biomed Imaging Interv J 7: e5, 2011. 